\title{
Análise do comportamento espectral da água do Rio Doce, Brasil, mediante o rompimento da barragem de sedimentos da empresa SAMARCO
}

\section{Analysis of the spectral behavior of the water of the Doce river, Brazil, through the rupture of the sediment dam of SAMARCO}

Laura Thebit-Almeida ${ }^{1}$, Felipe Bernardes-Silva², Micael de Souza-Fra$\mathrm{ga}^{3}$, Rayssa Balieiro-Ribeiro ${ }^{4}$, Maria Camila Alves-Ramos ${ }^{5}$, Roberto Avelino-Cecílio ${ }^{6}$

Thebit-Almeida, L; Bernardes-Silva, F; Souza-Fraga, M; Balieiro-Ribeiro, R; Alves-Ramos, M.C; Avelino-Cecílio, R. Análise do comportamento espectral da água do Rio Doce, Brasil, mediante o rompimento da barragem de sedimentos da empresa SAMARCO. Tecnología en Marcha. Vol. 32, Especial. XIII CLIA. Abril 2019. Pág 36-42.

DOI: https://doi.org/10.18845/tm.v32i7.4257

1 Estudante de doutorado. Universidade Federal de Viçosa, Departamento de Engenharia Agrícola, Brasil. Correio eletrônico: I.thebit@gmail.com,

2 Estudante de doutorado. Universidade Federal de Viçosa, Departamento de Engenharia Agrícola, Brasil. Correio eletrônico: felipe.bernardes.ufv@gmail.com

3 Estudante de doutorado. Universidade Federal de Viçosa, Departamento de Engenharia Agrícola, Brasil. Correio eletrônico: micaelfraga@gmail.com

4 Estudante de doutorado. Universidade Federal de Viçosa, Departamento de Engenharia Agrícola, Brasil. Correio eletrônico: rayssabalieiro@gmail.com

5 Estudante de doutorado. Universidade Federal de Viçosa, Departamento de Engenharia Agrícola, Brasil. Correio eletrônico: mcamilaaramos@gmail.com

6 Professor. Universidade Federal do Espírito Santo, Brasil. Correio eletrônico: roberto.cecilio@ufes.br 


\section{Palavras-chave \\ Reflectância; Turbidez; Sensoriamento Remoto.}

\section{Keywords}

Reflectance, Turbidity, Remote Sensing.

\section{Resumo}

A Barragem de Fundão, localizada na Bacia Hidrográfica do Rio Doce, Brasil, destinava-se ao acúmulo de sedimentos das atividades de extração de minério de ferro da empresa SAMARCO. Esta barragem rompeu-se no dia 5 de novembro de 2015, causando enorme desastre ambiental em todos os cursos d'agua à jusante, até atingir o Oceano Atlântico. A lama de rejeitos atingiu - Rio Doce e alterou a qualidade da água para captação e consumo em diversos municípios, sendo um desses o de Governador Valadares (MG). Sabe-se que, dentro das aplicações do sensoriamento remoto para a engenharia, a análise visual e do comportamento espectral dos alvos auxilia a tomada de decisão para a recuperação e o manejo de bacias hidrográficas. Desta forma, analisou-se o comportamento espectral da água e do NDWI (Normalized Difference Water Index) de seis imagens do Landsat 8 para o município de Governador Valadares, sendo duas antes e quatro após o rompimento da barragem (datas 05/08/2014; 25/09/2015; 12/11/2015, 16/02/2016; 10/08/2016 e 10/06/2017). Em escala de 0-1, o pico de reflectância da água antes ao ocorrido (05/08/2014) era da banda do verde, de 0,048. A imagem referente ao dia 12/11/2015, logo após o rompimento, apresentou maior reflectância em todas as bandas, com maior pico na infravermelho próximo $(0,48)$, banda esta correspontente à resposta dos alvos sólidos suspensos na água e vegetação, bem como elevação do NDWI. A reflectância foi reduzida após 8 meses do desastre, entretanto em nenhuma data analisada o comportamento espectral equivaleu-se ao observado antes do desastre ambiental.

\section{Introdução}

A bacia do Rio Doce está localizada na região Sudeste do Brasil, com área de drenagem de $83.465 \mathrm{~km}^{2}$, sendo que 86\% encontra-se no estado de Minas Gerais (MG) e 14\% no Espírito Santo (ES)[1]. O regime do Rio Doce, é caracterizado por alternância entre cheias de verão e vazantes acentuadas na estiagem. O clima no interior da bacia é considerado tropical úmido [2], com início da estação chuvosa em novembro [1].

O vale do Rio Doce começou a fazer parte da temática "emancipação nacional" a partir da Revolução de 1930. Em que foi considerado estratégico para a industrialização, com instalação de ferrovias, rodovias, siderúrgicas e, principalmente, empresas mineradoras [1]. Uma das atividades que promoveu mudanças no uso do solo e, consequentemente, na qualidade da água do Rio Doce foi a mineração. O impacto dessa atividade é relacionado, num primeiro momento, com desmatamento para abertura das minas e, posteriormente, com o descarte dos rejeitos, que, comumente são direcionados para barragens.

A barragem de Fundão era uma barragem de sedimentos proveniente da extração de minério de ferro, pertencente a empresa SAMARCO, localizada na cidade de Mariana, Minas Gerais, inserida na Bacia do Rio Doce. Seu rompimento, ocorrido no dia 5 de novembro de 2015, promoveu enorme desastre ambiental devido a sua pluma de rejeitos. A lama alastrou $663 \mathrm{~km}$ nos rios Gualaxo do Norte, Carmo e Doce até chegar na foz do último, onde adentrou $80 \mathrm{~km}^{2}$ ao mar [1], [3]. 
Os sólidos suspensos que se espalharam pelo rio promoveram aumento na turbidez da água e sua contaminação, afetando a captação em diversos municípios, tais como Governador Valadares (MG) e Colatina (ES) [3]. A contaminação da água e redução da luminosidade é prejudicial à vida aquática, prejudicando diversas espécies, principalmente pela redução do oxigênio disponível. Diante desse cenário, o sensoriamento remoto surge como uma possível forma de complementação dos programas de monitoramento de qualidade da água e estudos hidrológicos [4] [5], auxiliando na tomada de decisão para a recuperação e o manejo de uma bacia hidrográfica.

Sedimentos em suspensão, pigmentos fotossintetizantes, matéria orgânica dissolvida e as moléculas de água, em si, são os principais agentes que regem as propriedades ópticas inerentes da água e, portanto, são chamados de constituintes opticamente ativos [6]. Este conhecimento aliado ao desenvolvimento de sensores, capazes de registrar com detalhe as propriedades espectrais dos alvos na superfície terrestre, e de métodos de análise, que permitem a extração de informações cada vez mais específicas e precisas, têm aperfeiçoado o estudo da composição da água por meio de técnicas de sensoriamento remoto [7].

Desta forma, o intuito deste trabalho foi obter e analisar, por imagem do satélite Landsat 8, o comportamento espectral da água do Rio Doce, no município de Governador Valadares, antes e após o desastre do rompimento da barragem de sedimento de Fundão até o ano de 2017.

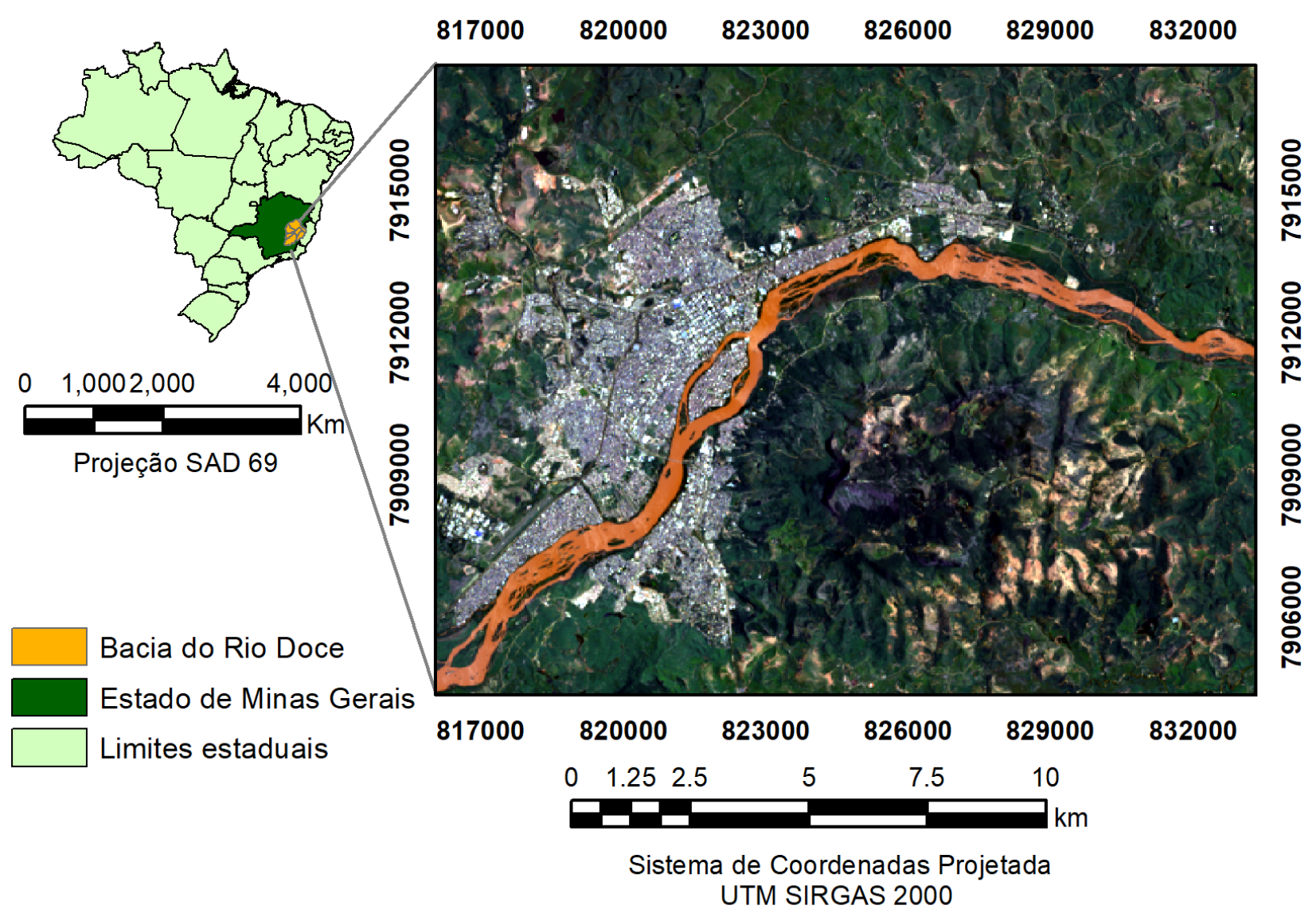

Figura 1. Localização do Rio Doce no município de Governador Valadares -MG/Brasil. 


\section{Metodologia}

Para o desenvolvimento do estudo foram obtidas imagens do satélite Landsat 8, sensor OLI, do Rio Doce na região do município de Governador Valadares-MG (figura 1). Duas imagens referentes aos dias 05/08/2014 e 25/09/2015, antecedentes ao desastre, e quatro imagens, referentes aos dias 12/11/2015, 16/02/2016, 10/08/2016 e 10/06/2017, posteriores ao mesmo. Tais imagens foram adquiridas gratuitamente na plataforma online United States Geologycal Service (USGS), com correção atmosférica e reflectância da superfície. O procedimento de projeção das imagens para sistema de projeção UTM Sirgas 2000, zona 23S se deu no software ArcGis 10.5.1. Em seguida, na plataforma Spring 5.1 do INPE, foram coletados dados dos números digitais de cada imagem, sobre os mesmos pontos, nas bandas: 1 - Aerosol Costeiro

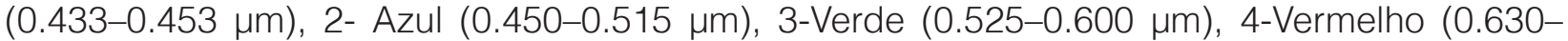
$0.680 \mu \mathrm{m}), 5$ - Infravermelho próximo $(0.845-0.885 \mu \mathrm{m}), 6$ - Infravermelho de Ondas Curtas1 (1.560-1.660 $\mu \mathrm{m})$ e 7- Infravermelho de Ondas Curtas $2(2.100-2.300 \mu \mathrm{m})$.

O índice analisado no estudo foi o NDWI (Normalized Difference Water Index), de acordo com a equação proposta por Gao (1996). Para tanto, utilizou-se o comprimento de onda que se refere a banda do Infravermelho Próximo (NIR) e da banda do Infravermelho de Ondas Curtas 1(SWIR1), bandas 5 e 6, respectivamente.

\section{Resultados e discussão}

Corpos d'água quando limpos e profundos apresentam alta absorção em quase todos os comprimentos de onda, com exceção apenas de um pico próximo ao comprimento 0.500 $\mu \mathrm{m}$, referente às bandas do azul e do verde. As bandas 4 e 5 estão relacionadas à resposta espectral do vermelho $(R)$ e ao NIR, e a banda 6 ao SWIR1. Na faixa do NIR a vegetação apresenta alta reflectância devido a sua estrutura, logo, corpos de água com alta reflectância na banda 5 apresentam excesso de algas devido, provavelmente, à eutrofização ou vegetação aquática. Água com elevado teor de sólidos suspensos refletem no R, NIR e SWIR1[8].

Observando a figura 2, a imagem referente ao dia 5/08/2014, anterior ao desastre, mostra que o comportamento espectral da água se aproxima mais das condições normais desse alvo, isto é, maior absorção e menor reflectância (pouca reflectância no comprimento de onda de 0,55 m, referente ao verde), indicando uma melhor qualidade da mesma, isto é, com menor quantidade de sólidos em suspensão que promovem aumento da reflectância. Esse comportamento pode ser definido como comportamento espectral real do Rio Doce no período seco, caracterizado pela decantação das partículas no leito do rio e baixa turbidez da água. No dia 25/09/2015, anterior ao desastre, a reflectância é superior em relação ao ano de 2014, o que pode ser devido ao fato de ter chovido anteriormente a aquisição da imagem. A ocorrência do escoamento superficial, que transporta sedimentos para o rio juntamente ao aumento do fluxo de correnteza, eleva a turbidez do curso e, consequentemente, sua reflectância.

A imagem correspondente ao dia 12/11/2015, posterior ao desastre, quando há alto teor de sólidos em suspensão na água, foi a que apresentou maior reflectância, principalmente no comprimento de onda do vermelho $(0,66 \mu \mathrm{m})$ e do $\mathrm{NIR}(0,86 \mu \mathrm{m})$, sendo observado um aumento de cerca de 23 vezes em relação a imagem de 2014 para a reflectância da banda NIR. Isso indica elevação de partículas e aumento da turbidez da água. Em 16/02/2016, dois meses após o rompimento do barramento, houve redução pela metade da reflectância no NIR em comparação com o dia 12/11/2015. Em 10/08/2016, período seco, bem como 27/06/2017, a redução da reflectância é ainda maior, entretanto superior ao valor obtido para 2014, antes do rompimento da barragem de Fundão. Esse resultado mostra a recuperação da turbidez da água do rio ao longo do tempo. 
Observa-se na figura 3, que o NDWI aumentou de 0,09 (25/09/2015) para 0,36 na data de 12/11/2015, logo em seguida ao desastre, apresentando pico máximo de 0,835 em 16/02/2016. Em 10/08/2016, é observado uma redução para 0,29, assim como em 27/06/2017 $(0,14)$, voltando a um nível próximo ao anterior ao rompimento, nas datas de 05/08/2014 $(0,19)$ e 25/09/2015 (0,06).

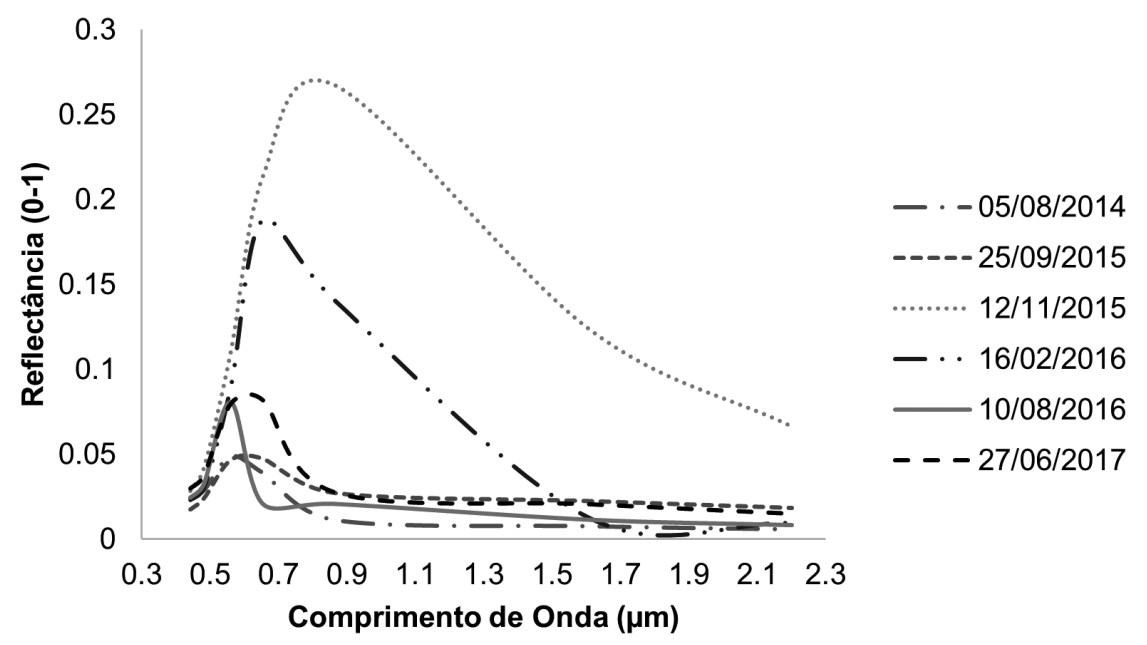

Figura 2. Comportamento espectral da água do Rio Doce.

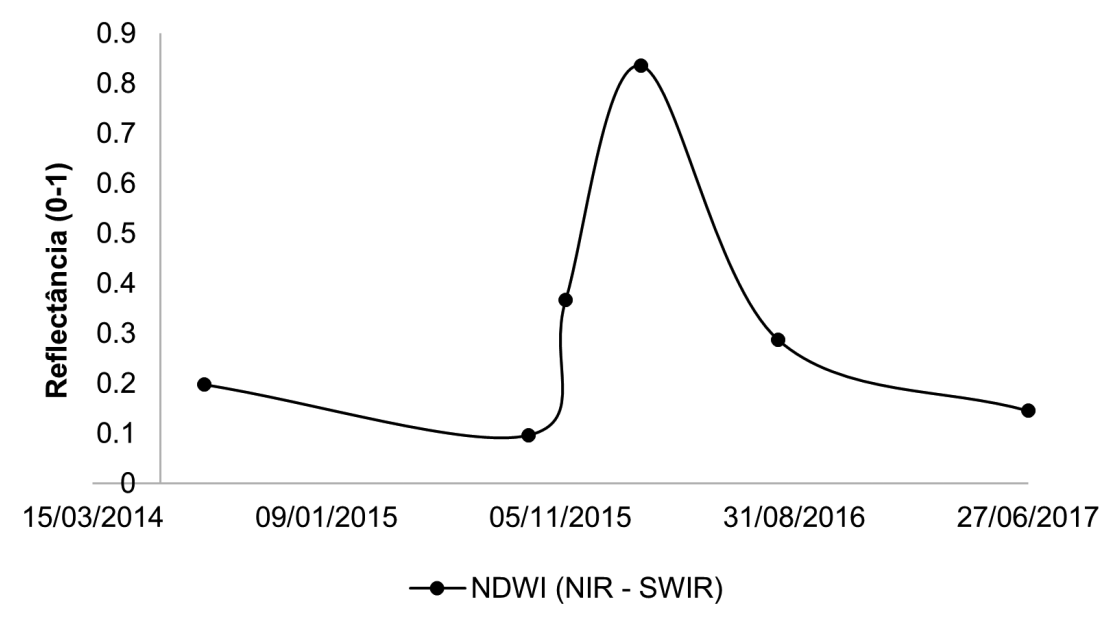

Figura 3. NDWI da água do Rio Doce para diferentes datas.

A figura 4 apresenta todas as imagens obtidas da área de estudo em que as bandas 4, 3 e 2 estão representadas na cor real dos canais vermelho, verde e azul, respectivamente. Nos dias 12/11/2015 e 16/02/2015, a cor refletida pelo rio é na tonalidade marrom, corroborando com o que foi discutido anteriormente.

Nota-se que o desastre do rompimento da barragem de Fundão conduziu a um elevado teor de sedimentos de rejeitos de mineração para o Rio Doce na região de Governador Valadares. O comportamento espectral da água mostrou que houve elevada reflectância nas bandas do vermelho, infravermelho próximo e infravermelho de ondas curtas, o que se deve aos sólidos 
em suspensão presentes na água, que reduzem a sua translucidez e absorbância. A redução da translucidez inibe a entrada de luz solar na água, bem como reduz a quantidade de oxigênio dissolvido, o que promoveu redução da vida aquática (figura 4).

As imagens mostram que após 9 meses do rompimento da barragem, o comportamento espectral da água estava próximo ao observado nas datas que antecedem esta ocorrência. Porém, em 2017, ainda se observa alta reflectância na banda do verde em comparação às imagens anteriores ao desastre, o que indica que ainda há uma maior concentração de sólidos em suspensão no Rio Doce, especificamente na cidade de Governador Valadares. Outro fato a ser levado em consideração é que não somente o retorno da translucidez do rio indica a recuperação do mesmo. Do material lançado, em relação aos materiais inorgânicos, apresentou elevado teor de óxido de ferro, manganês e sílica [9], logo, para maiores inferências é necessária uma análise química espaço-temporal da água.

Dos casos de rompimento de barragem de rejeito, foram registrados sete apenas no estado de Minas Gerais desde 1986 [8]. Esse fato evidencia a necessidade de estudos técnicos mais aprofundados para avaliação de impactos ambientais decorrentes da implementação e operação deste tipo de empreendimento, assim como, um monitoramento das condições de funciomento dos mesmos.
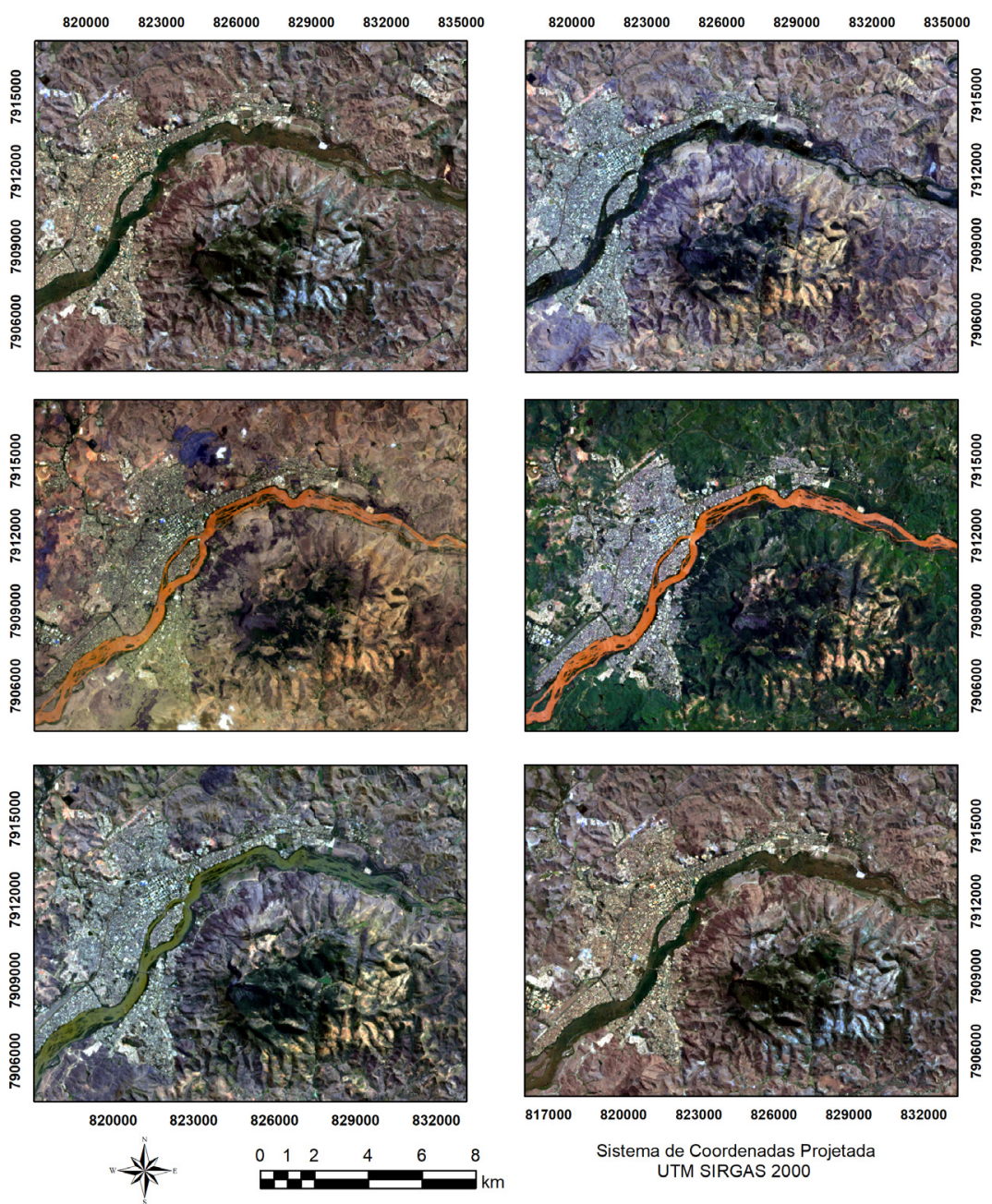

Figura 4. Composição colorida RGB 432 da área de estudo. 


\section{Conclusão}

Foi observada elevada reflectância e aumento no valor do índice NDWI para as imagens com datas posteriores e mais próximas da ocorrência do desastre (12/11/2015 e 16/02/2016), o que indica uma maior concentração de sólidos em suspensão nesse período. Em 10/08/2016 e 27/06/2017 os valores de reflectância e do NDWI diminuíram, indicando uma redução da concentração dos sólidos em suspensão da água do Rio Doce.

\section{Referências}

[1] H. S. Espindola, "O rio Doce e a emancipação da economia nacional (Brasil)", Hist. Ambient. Latinoam. Caribeña HALAC Rev. Solcha, vol. 5, n 1, 2015.

[2] A. L. N. Coelho, "Bacia hidrográfica do Rio Doce(MG/ES):uma análise socioambiental integrada", Rev. Geogr., vol. 7, p. 131-146, out. 2009.

[3] Samarco, "Entenda o rompimento", 2017. [Online]. Disponível em: https://www.samarco.com/rompimento-defundao/.

[4] M. T. de S. Machado e G. M. de M. Baptista, "Sensoriamento remoto como ferramenta de monitoramento da qualidade da água do Lago Paranoá (DF)”, Eng. Sanit. E Ambient., vol. 21, n 2, p. 357-365, jun. 2016.

[5] N. P. A. Vieira, F. B. Silva, C. R. Nascimento, E. O. Vieira, e M. S. Fraga, "Soil Losses in the Cedro River Basin/ MG Based on the Sensor TM/LANDSAT-5 Images", Nativa, vol. 4, n 5, p. 261-270, out. 2016.

[6] C. M. Rudorff, E. M. L. M. Novo, L. S. Galvão, e W. Pereira Filho, "Análise derivativa de dados hiperespectrais medidos em nível de campo e orbital para caracterizar a composição de águas opticamente complexas na Amazônia", Acta Amaz., vol. 37, n² 2, p. 269-280, jun. 2007.

[7] V. Méléder, L. Barillé, P. Launeau, V. Carrère, e Y. Rincé, "Spectrometric constraint in analysis of benthic diatom biomass using monospecific cultures", Remote Sens. Environ., vol. 88, n 4, p. 386-400, dez. 2003.

[8] R. de O. Borges, F. F. Camargo, F. Campagnoli, e M. Bayer, "Aplicação do índice da diferença normalizada da água (NDWI) na delimitação de fluxos de sedimentos em suspensão no Rio Araguaia: avaliação das imagens Landsat 8 para o", in Anais XVII, João Pessoa - PB - Brasil, 2015.

[9] C. M. de Freitas, M. A. da Silva, e F. C. de Menezes, "O desastre na barragem de mineração da Samarco: fratura exposta dos limites do Brasil na redução de risco de desastres", Ciênc. E Cult., vol. 68, n 3, p. 25-30, set. 2016. 